\title{
Medo, ansiedade e controle relacionados ao tratamento odontológico
}

\section{Fear, anxiety and control related to dental treatment}

\author{
Kira Anayansi SINGH* \\ Antonio Bento Alves de MORAES** \\ Gláucia Maria BOVI AMBROSANO***
}

\begin{abstract}
SINGH, K. A.; MORAES, A. B. A. de; BOVI AMBROSANO, G. M. Medo, ansiedade e controle relacionados ao tratamento odontológico. Pesq Odont Bras, v. 14, n. 2, p. 131-136, abr./jun. 2000.
\end{abstract}

\begin{abstract}
Nosso objetivo foi avaliar medo, ansiedade e controle relacionados ao tratamento odontológico. Os sujeitos foram 364 crianças da faixa etária de 7 a 13 anos. Três questionários com questões de múltipla escolha foram aplicados em grupos de 10 crianças. O primeiro questionário destinou-se à avaliação do medo ao tratamento odontológico e outras situações. Foi traduzido e adaptado do "Child's Fear Survey Schedule"9 e contém 15 itens. O segundo questionário contém 20 itens relacionados as situações potencialmente produtoras de ansiedade. Foi traduzido e adaptado do "State Trait Anxiety Inventory for Children" ${ }^{16}$. O terceiro questionário contém 40 itens sendo 20 relacionados ao controle percebido e 20 ao controle desejado e foi traduzido e adaptado do "Child Dental Control Assessment"19. Em relação ao medo e ansiedade, a média dos escores foi mais elevada para o sexo feminino do que para o sexo masculino $(\mathrm{P}<0,05)$. Em relação a idade, as crianças da faixa de 11 a 13 anos revelaram-se em média mais temerosas que as de 7 a 9 anos. Quanto ao controle percebido as crianças mais novas percebem mais controle do que as mais velhas. Em geral os dados indicam que valores mais altos de medo relacionam-se com valores mais baixos de controle percebido. As crianças que tinham realizado tratamento odontológico com anestesia mostraram-se mais temerosas do que aquelas que não foram submetidas a anestesia, o que permite inferir que a etiologia de medo pode relacionar-se a esse procedimento que pode envolver a percepção de incontrolabilidade.
\end{abstract}

UNITERMOS: Medo; Ansiedade; Odontopediatria.

\section{INTRODUÇÃO}

O medo é parte do desenvolvimento infantil. Em geral é transitório e não produz grandes perturbações na vida diária da criança. Embora a capacidade de vivenciar o medo, seja uma função biológica inata, respostas de medo a certos objetos e situações são em grande parte adquiridas através da aprendizagem.

$\mathrm{Na}$ verdade, as experiências com medos apropriados à idade ajudam a criança a desenvolver habilidades de enfrentamento. No entanto, muitos medos infantis inicialmente normais podem persistir por longos períodos e produzir diversos problemas para a criança e para sua família. Assim pode acontecer em relação ao medo do tratamento odontológico. Esse medo pode ter diversas origens sendo que as mais freqüentes são as experiências vividas pela própria criança no tratamento odontológico. Outras podem ser transmiti- das à criança por pessoas diretamente no meio familiar ou mais indiretamente através dos meios de comunicação ${ }^{13,18}$.

Algumas evidências indicam que o medo do tratamento odontológico começa na infância mas pouco se conhece como esses medos (e os comportamentos de esquiva subseqüentes) se desenvolvem $^{3,12}$.

A ansiedade, por outro lado, é entendida como uma resposta à situações nas quais a fonte de ameaça ao indivíduo não está bem definida, é ambígua ou não está objetivamente presente ${ }^{11}$.

Segundo PESSOTTI ${ }^{15}$ (1978), a ansiedade implica na ocorrência de uma condição aversiva ou penosa, algum grau de incerteza ou dúvida e alguma forma de impotência do organismo em uma dada conjuntura.

Outro fator que muitos pesquisadores consideram de importância é o sentimento de controle

\footnotetext{
* Aluna do Curso de Pós-Graduação (Doutorado) em Odontopediatria da Faculdade de Odontologia de Araraquara da UNESP.

** Professor Titular; *** Professora Doutora - Departamento de Odontologia Social da Faculdade de Odontologia de Piracicaba da UNICAMP, Bolsistas do Conselho Nacional de Desenvolvimento Científico e Tecnológico (CNPq).
} 
SINGH, K. A.; MORAES, A. B. A. de; BOVI AMBROSANO, G. M. Medo, ansiedade e controle relacionados ao tratamento odontológico.

Pesq Odont Bras, v. 14, n. 2, p. 131-136, abr./jun. 2000.

uma vez que o desejo de exercer controle sobre o ambiente parece ser uma necessidade básica demonstrada em vários estudos ${ }^{3,19}$.

LOGAN et al. ${ }^{8}$ (1991) aponta a falta de controle como um fator de risco para medos diretamente condicionados. $\mathrm{O}$ fator controle refere-se a situações em que o indivíduo percebe que tem em seu repertório condutas que podem diminuir ou eliminar um evento aversivo presente. THOMPSON ${ }^{17}$ (1981) sugeriu que a percepção de controle afeta as respostas de ansiedade. Entretanto, até bem recentemente, as pesquisas não têm apresentado evidências conclusivas sobre como o controle afeta a ansiedade ou o medo.

WEINSTEIN et al. ${ }^{19}$ (1996) estuda a avaliação do controle da criança no tratamento odontológico, em uma situação específica na qual o autor identifica as estratégias de controle que crianças de idade escolar utilizam quando visitam o dentista. O primeiro estudo foi realizado com 180 crianças e os dados foram obtidos em sala de aula onde foram aplicados três questionários: de medo, de ansiedade e de controle. A análise estatística indicou cinco categorias de respostas: controle mediado pelo dentista, enfrentamento ativo, afastamento cognitivo, tranqüilização e fuga. As categorias que tiveram escores mais altos foram tranqüilização e controle mediado pelo dentista.

Para o segundo estudo foram aplicados os mesmos questionários e foram oferecidos dispositivos sinalizadores para que os sujeitos pudessem exercer controle sobre a situação. A criança apertava um botão na cadeira odontológica que acionava o dispositivo sinalizador, quando estava tudo bem, a cor escolhida deveria ser verde. Quando a criança sentia-se preocupada a cor deveria ser o amarelo e quando ela queria que o dentista interrompesse o tratamento escolhia a cor vermelha. Os resultados indicaram que a idade e a percepção da aversividade dos procedimentos clínicos, são variáveis importantes para o uso da sinalização. Crianças mais novas percebem o sinalizador como um mecanismo de enfrentamento. Crianças mais velhas, não o consideram de grande ajuda. Entretanto, para todas as crianças, o autor sugere que tratamentos invasivos e a percepção da aversividade levam a criança a utilizar o dispositivo sinalizador como forma de fuga.

A etiologia de medos clínicos severos parece estar relacionada à idade e ao condicionamento direto na presença de dor e vulnerabilidade ${ }^{14}$. O medo do tratamento odontológico torna-se cíclico no sentido de que quando a patologia dentária não é regularmente tratada pelos serviços preventivos ela desencadeia a utilização de serviços odontológicos curativos ou de emergência inerentemente invasivos e dolorosos. Tais tratamentos exacerbam ou produzem medo e comportamento de esquiva a futuros tratamentos.

Esta pesquisa estuda o medo, ansiedade e controle em função do sexo e a faixa etária e parte do pressuposto de que variáveis cognitivas, como a percepção de controle, podem moderar a aversividade do tratamento em crianças.

\section{MATERIAL E MÉTODO}

Em 364 crianças (sete a treze anos de idade) de ambos os sexos foram aplicados os questionários de avaliação do medo, ansiedade e controle. Os sujeitos pertenciam a uma escola da periferia de Paulínia, SP e participavam de um programa de atendimento odontológico à comunidade. Aleatoriamente eram formados grupos de 10 alunos que acompanhavam a pesquisadora para outra sala onde era realizada a aplicação dos questionários. O pesquisador lia as questões e as crianças assinalavam suas respostas, caso houvessem dúvidas referentes à compreensão, eram esclarecidas e registradas.

Os instrumentos originais em inglês de medo, ansiedade e controle foram traduzidos e adaptados seguindo-se os seguintes passos: uma pessoa com dominio de ambas as linguas (português e inglês) fez a primeira tradução dos instrumentos para o português; uma segunda pessoa com domínio de ambas as línguas, fez a versão do português para o inglês sem ter acesso aos originais. A pessoa responsável pelo projeto que também domina ambas línguas fez a comparação das traduções e realizou as alterações necessárias. Em seguida os questionários passaram por um controle de qualidade preliminar e posteriormente foram submetidos à avaliação formativa e somativa. O controle de qualidade preliminar consistiu numa revisão do material elaborado, antes de sua primeira aplicação ${ }^{1,4}$. Neste caso um especialista no conteúdo verificou sua precisão e abrangência e um especialista em avaliação educacional verificou a disposição e formato dos itens de cada instrumento de avaliação.

A avaliação formativa tem como objetivo melhorar e aperfeiçoar o material conduzindo a reformulação quando necessário. Foram selecionados ao acaso quarenta alunos de uma escola pública de Piracicaba, sendo vinte da faixa de oito a nove anos de idade e vinte da faixa de doze a treze e os instrumentos foram aplicados para ambos os grupos. 
SINGH, K. A.; MORAES, A. B. A. de; BOVI AMBROSANO, G. M. Medo, ansiedade e controle relacionados ao tratamento odontológico.

Pesq Odont Bras, v. 14, n. 2, p. 131-136, abr./jun. 2000.

Após essa a avaliação realizou-se uma alteração no questionário de medo; no segundo instrumento não foi realizada nenhuma alteração e no terceiro instrumento foram eliminadas duas questões. Depois destes procedimentos procedeu-se a avaliação somativa na qual os 364 indivíduos preencheram três instrumentos. O primeiro instrumento destinou-se à avaliação do medo da criança ao tratamento odontológico. Ele foi traduzido e adaptado do "Child's Fear Survey Schedule", e compõe-se de 15 itens relacionados as situações que a criança possa temer, por exemplo, em relação a "dentista", "médico", "injeção" etc. Os indivíduos indicaram seu nivel de medo numa escala com cinco alternativas de respostas que variava de "nenhum medo" a "um medo intenso". O segundo instrumento destinou-se à avaliação da ansiedade apresentada pela criança e foi traduzido e adaptado do "State Trait Anxiety Inventory for Children"16. Compunha-se de 20 itens relacionados às situações potencialmente produtoras de ansiedade e pretendia medir de maneira generalizada a ansiedade da criança. Entre os itens pode-se citar "eu me preocupo com os meus pais", "eu me preocupo com as coisas que possam acontecer" etc. Cada item tinha três alternativas de escolha: "quase nunca", "algumas vezes" e "freqüentemente". O terceiro instrumento destinou-se à avaliação da variável controle e foi traduzido e adaptado do "Child Dental Control Assessment" ${ }^{19}$. Neste aparece, inicialmente, uma série de exercícios que foram realizados com as crianças para facilitar a compreensão e o correto preenchimento da ficha. O instrumento consta de uma lista de 40 itens. Os 20 itens indicados como A referem-se aos eventos que aconteceram na última sessão de tratamento odontológico e procuram identificar a variável "controle percebido". Entre os itens pode-se citar "na última sessão o dentista me disse o que ia acontecer", "na última sessão o dentista me disse que tudo daria certo" etc. Os 20 itens indicados como B referem-se ao que a cri-

TABELA 1 - Escore médio do medo total, controle percebido total, controle desejado total e ansiedade total, em função do sexo da criança.

\begin{tabular}{c|c|c|c|c}
\hline \hline Sexo & Medo & $\begin{array}{c}\text { Controle } \\
\text { percebido }\end{array}$ & $\begin{array}{c}\text { Controle } \\
\text { desejado }\end{array}$ & Ansiedade \\
\hline $\mathrm{F}$ & $29,1 \mathrm{~A}$ & $22,6 \mathrm{~A}$ & $32,0 \mathrm{~A}$ & $37,7 \mathrm{~A}$ \\
\hline $\mathrm{M}$ & $26,6 \mathrm{~B}$ & $23,4 \mathrm{~A}$ & $32,9 \mathrm{~A}$ & $36,8 \mathrm{~B}$ \\
\hline \hline
\end{tabular}

Médias seguidas de letras distintas na vertical indicam diferenças significativas pelo teste $t$ de Student $(\mathrm{P}<0,05)$. ança deseja que aconteça na próxima sessão e procuram identificar a variável "controle desejado". Pode-se citar alguns itens: "na próxima sessão eu quero que o dentista responda todas as minhas perguntas", "na próxima sessão eu quero que o dentista me diga que me comportei bem".

Para a análise dos dados obtidos após a aplicação dos três instrumentos, utilizou-se estatística descritiva e o teste $t$ de Student.

\section{RESULTADOS}

Na Tabela 1 observa-se que para o Instrumento de Avaliação do Medo, a média dos escores foi mais alta para o grupo do sexo feminino com 29,1 do que do sexo masculino que foi $26,6(\mathrm{P}<0,05)$ o que mostra resultados semelhantes aos encontrados em outros estudos onde as mulheres revelam possuir mais medo que os homens ${ }^{12}$.

Em relação a ansiedade as médias também mostraram-se diferentes em função do sexo, sendo que as meninas tiveram um valor médio mais elevado que os meninos $(\mathrm{P}<0,05)$ revelando-se mais ansiosas.

Para o controle percebido e desejado os dados não revelaram diferença estatisticamente significantes.

Na Tabela 2 observa-se que o valor médio de medo para o grupo de menor idade foi de 26,3 e para o grupo de maior idade foi $33,3(\mathrm{P}<0,05)$ o que revela que os sujeitos de maior idade são em média mais temerosos que os sujeitos do grupo de menor idade.

No Instrumento de Avaliação da Ansiedade, a média dos escores foi igual para ambos os grupos, o que revela que a idade não influenciou o grau de ansiedade apresentada pelos sujeitos.

Quanto ao controle percebido, o valor médio foi maior para o grupo de menor idade $(24,5)$ e o grupo de maior idade teve uma média de $17,9(\mathrm{P}<0,05)$. Esses dados revelam que as crianças mais novas percebem mais controle que as mais velhas, e têm

TABELA 2 - Escore médio do medo, controle percebido, controle desejado e ansiedade, em função da faixa etária da criança.

\begin{tabular}{c|c|c|c|c}
\hline \hline $\begin{array}{c}\text { Idade } \\
\text { (anos) }\end{array}$ & Medo & Ansiedade & $\begin{array}{c}\text { Controle } \\
\text { percebido }\end{array}$ & $\begin{array}{c}\text { Controle } \\
\text { desejado }\end{array}$ \\
\hline $7-9$ & $26,3 \mathrm{~B}$ & $37,1 \mathrm{~A}$ & $24,5 \mathrm{~A}$ & $34,5 \mathrm{~A}$ \\
\hline $11-13$ & $33,3 \mathrm{~A}$ & $37,9 \mathrm{~A}$ & $17,9 \mathrm{~B}$ & $25,5 \mathrm{~B}$ \\
\hline \hline
\end{tabular}

Médias seguidas de letras distintas na vertical indicam diferenças significativas pelo teste $t$ de Student $(\mathrm{P}<0,05)$. 
SINGH, K. A.; MORAES, A. B. A. de; BOVI AMBROSANO, G. M. Medo, ansiedade e controle relacionados ao tratamento odontológico.

Pesq Odont Bras, v. 14, n. 2, p. 131-136, abr./jun. 2000.

TABELA 3 - Escore médio do medo, controle percebido, controle desejado e ansiedade para as crianças com idade entre 7 e 9 anos e 11 a 13 anos, que receberam ou não anestesia.

\begin{tabular}{c|c|c|c|c|c}
\hline \hline Anestesia & Idade & Medo & Ansiedade & $\begin{array}{c}\text { Controle } \\
\text { percebido }\end{array}$ & $\begin{array}{c}\text { Controle } \\
\text { desejado }\end{array}$ \\
\hline Não & $7-9$ & $25,9 \mathrm{~B}$ & $33,5 \mathrm{~B}$ & $19,8 \mathrm{~A}$ & $35,5 \mathrm{~A}$ \\
\hline Sim & $7-9$ & $29,2 \mathrm{~A}$ & $35,5 \mathrm{~A}$ & $19,9 \mathrm{~A}$ & $34,5 \mathrm{~A}$ \\
\hline Não & $11-13$ & $24,9 \mathrm{~B}$ & $34,9 \mathrm{~B}$ & $19,7 \mathrm{~A}$ & $26,9 \mathrm{~A}$ \\
\hline Sim & $11-13$ & $37,6 \mathrm{~A}$ & $38,9 \mathrm{~A}$ & $19,4 \mathrm{~A}$ & $28,2 \mathrm{~A}$ \\
\hline \hline
\end{tabular}

Médias seguidas de letras distintas na vertical indicam diferenças significativas dentro de cada idade pelo teste $t$ de Student $(\mathrm{P}<0,05)$.

menos medo. Já as crianças mais velhas percebem menos controle e são em média um pouco mais temerosas.

No controle desejado, as crianças de menor idade, tiveram um valor médio de 34,5 e no grupo de maior idade as crianças tiveram o valor médio de $25,5(\mathrm{P}<0,05)$ o que revela que o grupo de crianças mais novas desejam mais controle que o grupo de crianças mais velhas.

$\mathrm{Na}$ Tabela 3 observa-se dados de crianças de duas faixas etárias diferentes que receberam ou não anestesia. Em relação ao medo as crianças de 7 a 9 que receberam anestesia tiveram médias mais altas $(29,2)$ do que as que não receberam $(25,9)$ semelhante ao resultado do grupo de 11 a 13 onde as que receberam anestesia tiveram média de 37,6 , e as que não receberam, 24,9 , o que revela que são em média mais temerosas independentemente da faixa etária.

A ansiedade também revelou diferença sendo para as crianças mais novas as que receberam anestesia tiveram média de 35,5 revelando-se mais ansiosas do que as que não receberam que tiveram média de 33,5. Em relação as crianças maiores as que receberam anestesia tiveram média de 38,9 e as que não receberam tiveram média de 34,9 resultado similar ao anterior, onde as crianças que recebem anestesia são mais ansiosas.

Em relação ao controle percebido e desejado as médias não tiveram diferença significativa entre os grupos avaliados.

As meninas se mostraram mais temerosas que os meninos. A média das meninas e dos meninos que receberam ou não anestesia, revelou diferença significativa, sendo que em relação ao medo, as meninas que receberam anestesia tiveram valor médio de 35,7 significativamente maior que as que não receberam 26,33. Para o sexo masculino, as
TABELA 4 - Escore médio do medo, controle percebido, controle desejado e ansiedade para as crianças com idade entre 11 e 13 anos, sexo masculino e feminino, que receberam ou não anestesia.

\begin{tabular}{c|c|c|c|c|c}
\hline \hline Anestesia & Sexo & Medo & Ansiedade & $\begin{array}{c}\text { Controle } \\
\text { percebido }\end{array}$ & $\begin{array}{c}\text { Controle } \\
\text { desejado }\end{array}$ \\
\hline Não & Fem. & $26,3 \mathrm{~B}$ & $32,7 \mathrm{~B}$ & $20,1 \mathrm{~A}$ & $32,3 \mathrm{~A}$ \\
\hline Sim & Fem. & $35,7 \mathrm{~A}$ & $38,1 \mathrm{~A}$ & $20,1 \mathrm{~A}$ & $30,3 \mathrm{~A}$ \\
\hline Não & Masc. & $25,2 \mathrm{~B}$ & $34,1 \mathrm{~B}$ & $32,7 \mathrm{~A}$ & $32,3 \mathrm{~A}$ \\
\hline Sim & Masc. & $32,1 \mathrm{~A}$ & $36,7 \mathrm{~A}$ & $38,1 \mathrm{~A}$ & $30,3 \mathrm{~A}$ \\
\hline \hline
\end{tabular}

Médias seguidas de letras distintas na vertical indicam diferenças significativas pelo teste $t$ de Student $(\mathrm{P}<0,05)$.

médias também foram significativamente diferentes sendo que os que receberam anestesia tiveram valor médio de medo de 32,1 e aqueles que não receberam anestesia 25,2.

Em relação a ansiedade, também as meninas se mostraram mais ansiosas que os meninos. Sendo que para o sexo feminino as que receberam anestesia tiveram valor médio de 38,1 e as que não receberam 32,7. Em relação aos meninos, também foram mais ansiosos aqueles que receberam anestesia, isto é, tiveram um valor médio de ansiedade de 36,7 e os que não receberam anestesia foi de 34,1 . No controle percebido e desejado os valores encontrados para os grupos que receberam e não receberam anestesia, não foram significativamente diferentes (Tabela 4).

\section{DISCUSSÃO}

Os resultados do estudo do medo de crianças em relação ao tratamento odontológico e os fatores de risco encontrados no presente trabalho são semelhantes aos encontrados em outros países como Estados Unidos e Singapura. No que se refere ao "medo odontológico" MILGROM et al. ${ }^{12}$ (1988), CUTHBERT; MELAMED ${ }^{3}$ (1982) e KLEINKNECHT et $a l^{5}{ }^{5}$ (1973) revelam que o medo é um problema reconhecido na população e que a maioria dos medos iniciam na infância, principalmente na fase escolar. Esta pesquisa baseada em respostas de 364 crianças da idade escolar (sete a treze anos) mostrou, que a idade e o sexo estão relacionadas com a percepção de medo.

Em relação ao sexo, indivíduos do sexo feminino apresentam escores mais altos do que indivíduos do sexo masculino, sendo semelhante aos resultados obtidos por MILGROM et al. ${ }^{12}$ (1988) e KLEINKNECHT et al. ${ }^{6}$ (1973). É interessante sali- 
SINGH, K. A.; MORAES, A. B. A. de; BOVI AMBROSANO, G. M. Medo, ansiedade e controle relacionados ao tratamento odontológico.

Pesq Odont Bras, v. 14, n. 2, p. 131-136, abr./jun. 2000.

entar o fato de que trabalhos realizados em culturas diferentes mostram resultados semelhantes. Isto sugere que as meninas podem ter maior facilidade para expressar suas emoções. No que se refere à idade observa-se que as crianças mais novas (7 a 9 anos) tiveram um valor médio de 26,3 com relação ao medo, e as crianças mais velhas (11 a 13 anos) tiveram um valor médio de 33,3 o que revela que as crianças mais velhas são, em média, um pouco mais temerosas. Pode-se sugerir que isso acontece com crianças mais velhas, porque estas vivenciaram um maior número de tratamentos odontológicos com a doença instalada e conseqüentemente serviços curativos invasivos e dolorosos. Tais serviços, provocando mais dor, levam a mais medo e ansiedade.

Para todas as crianças avaliadas independentemente da faixa etária e do sexo a ansiedade foi maior para aquelas que receberam anestesia quando comparadas com aquelas que não receberam anestesia. Este fato reafirma a hipótese de que tratamentos odontológicos invasivos sempre aumentam a ansiedade nas crianças.

MILGROM et $a l .{ }^{10}$ (1992) encontraram que adolescentes que tinham recebido tratamento odontológico na presença de dor ou desconforto estavam menos dispostos a retornar ao dentista do que aqueles que não tinham recebido tratamento invasivo ou desconfortável. WEINSTEIN et al. ${ }^{19}$ (1996) realizaram uma investigação que revelou que pacientes que precisavam de exodontias expressavam maior necessidade de ter controle do que aquelas não iriam realizar esse tipo de tratamento. Em relação à avaliação do controle, CORAH $^{2}$ (1973), LITT; SHAFER ${ }^{7}$ (1993), LOGAN et al. ${ }^{8}$ (1991), MILGROM; WEINSTEIN ${ }^{11}$ (1985), MILGROM et al. ${ }^{10}$ (1992), THOMPSON ${ }^{17}$ (1981) salientam a importância da percepção de controle sobre o ambiente e consideram-no até como uma necessidade básica. A falta de controle como um fator de risco para medos odontológicos diretamente condicionados tem sido identificado por pesquisadores e clínicos ${ }^{8,17}$.
Tem sido demonstrado que o exercício de controle afeta a tensão e o estresse e que a percepção de controle sobre estímulos aversivos reduz a aversividade do evento. Por outro lado, a diminuição do controle aumenta a tensão produzida pela situação estressante ${ }^{10}$.

Os resultados obtidos no presente trabalho demonstram que em relação ao controle percebido, o valor médio foi maior para o grupo de menor idade (7 a 9 anos), o que revela que as crianças mais novas percebem mais controle do que as mais velhas e têm menos medo; isto sugere que crianças mais velhas podem ter passado por experiências odontológicas prévias muito desagradáveis. Tais crianças percebem menos controle e são em média um pouco mais temerosas. Em relação ao controle desejado o grupo de crianças mais novas também manifestou desejo de maior controle do que o grupo de crianças mais velhas.

Experiências clínicas que utilizaram o controle percebido como uma estratégia efetiva de redução do estresse têm sido bem-sucedidas. Esses resultados sugerem que a percepção de controle possibilita uma melhora na capacidade de enfrentamento dos pacientes odontológicos em situações de medo e/ou ansiedade ${ }^{7}$.

\section{CONCLUSÕES}

1. Crianças com maior controle percebido apresentaram menor medo ao tratamento odontológico.

2. Crianças com experiência anterior de anestesia no tratamento odontológico revelaram-se mais temerosas do que as crianças que tinham feito tratamento sem anestesia.

3. As meninas mostraram-se mais temerosas e ansiosas do que os meninos.

4. Em relação a faixa etária as crianças de sete a nove anos de idade mostraram-se menos temerosas do que as crianças pertencentes a faixa de onze a treze anos. 
SINGH, K. A.; MORAES, A. B. A. de; BOVI AMBROSANO, G. M. Medo, ansiedade e controle relacionados ao tratamento odontológico.

Pesq Odont Bras, v. 14, n. 2, p. 131-136, abr./jun. 2000.

SiNGH, K. A.; MORAES, A. B. A. de; BOVI AMBROSANO, G. M. Fear, anxiety and control related to dental treatment.

Pesq Odont Bras, v. 14, n. 2, p. 131-136, abr./jun. 2000.

The aim of this study was to test fear, anxiety and control related to dental treatment. The subjects were 364 children with ages between 7 and 13 years. Three questionnaires with multiple choice questions were applied in groups of 10 children. The first instrument was the 15-item dental subscale from the Children's Fear Survey Schedule ${ }^{9}$ The subjects rated their level of fear on a 5-point scale. The second survey instrument was the 20-item subscale from the State Trait Anxiety Inventory for Children ${ }^{16}$. This measure was used to capture how anxious the child was, in general. The third instrument was the Child Dental Control Assessment ${ }^{19}$. It contained 20 items to assess perceived control and 20 items to assess desired control. The results of the survey indicated that dental fear and anxiety were slightly higher for females when compared with male subjects $(\mathrm{P}<0.05)$. Older children (11 to 13 years old) obtained higher fear scores than younger ones ( 7 to 9 years old). Concerning perceived control, the results indicate that younger children perceive more control than older ones. For desired control, the results indicate that younger children reported higher percentages than older ones. In this study, patients who had undergone anesthesia during treatment revealed higher fear scores when compared with those who had not. Dental fear etiology seems to be related to a procedure that may involve pain or lack of control.

UNITERMS: Fear; Anxiety; Pediatric dentistry.

\section{REFERÊNCIAS BIBLIOGRÁFICAS}

1. CHADWICK, C. Avaliação educacional. Rev Bras Teleeduc, v. 1, n. 4, p. 8-37, 1974.

2. CORAH, N. L. Effect of perceived control on stress reduction in pedodontic patients. J Dent Res, v. 52, p. 1261-1264, Nov./Dec. 1973.

3. CUTHBERT, M. I.; MELAMED, B. G. A screening device: children at risk for dental fears and management problems. J Dent Child, v. 49, p. 432-434, Nov./Dec. 1982.

4. DALEN, D.B.; MEYER, W. J. Manual de técnica de la investigación educacional. Buenos Aires : Editora Paidos, 1974.

5. KLEINKNECHT, R. A.; KLEPAC, R. K.; ALEXANDER, L. D. Origins and characteristics of fear of dentistry. J Am Dent Assoc, v. 86, p. 842-848, April 1973.

6. KLEINKNECHT, R. A.; McGLYNN, F. D.; THORNDIKE, R. et al. Factor analysis of the dental fear survey with cross-validation. J Am Dent Assoc, v. 108, p. 59-61, 1984.

7. LITT, M. D.; NYE, C.; SHAFER, D. Coping with oral surgery by self-efficacy enhancement and perceptions of control. J Dent Res, v. 72, p. 1237-1243, August 1993.

8. LOGAN, H. L.; BARON, R.; KEELEY, K. et al. Desired control and felt control as mediators of stress in a dental setting. Health Psychol, v. 10, p. 352-359, 1991.

9. MelameD, BG; LUMLey, M. A. Dental Subscale of the Children's Fear Survey Schedule (1988). In Hersen, M \& BELLACH, A S. (Eds.) Dictionary of behavioral assess- ment techniques. Oxford : Pergamom Press, p. 171-172.

10. MILGROM, P.; VIGNEHSA, H.; WEINSTEIN, P. Adolescent dental fear and control: prevalence and theoretical implications. Beh Res Ther, v. 30, p. 367-373, 1992.

11. MILGROM, P.; WEINSTEIN, P. Treating fearful dental patients. A patient management handbook. Seattle : Ed. Reston Pub., 1985, p. 3-6, 45-48.

12. MILGROM, P.; FISET, L.; MELNICK, S. et al. The prevalence and practice management consequences of dental fear in a major US city. J Am Dent Assoc, v. 116, p. 641-647, 1988.

13. MORAES, A. B. A.; GIL, I. A. A criança e o medo do tratamento odontológico. In: USBERTI, A. C. Odontopediatria clínica. 2. ed. São Paulo : Ed. Santos, p. 113-119, 1993.

14. OST, L. G. Acquisition of blood and injection phobia and anxiety response patterns in clinical patients. Beh Res Ther, v. 29, p. 323-332, 1991.

15. PESSOTTI, I. Ansiedade. São Paulo : Ed. Pedagógica e Universitária, 1978.

16. SPIELBERGER, C. D. STAIC-Preliminary Manual, Palo Alto, Consulting Psychologist Press, 1973.

17. THOMPSON, S. C. Will it hurt less if I can control it? A complex answer to a simple question. Psychol Bull, v. 90, p. 89-101, 1981.

18. WEINSTEIN, P. The effect of dentists' behaviors on fear-related behaviors in children. J Am Dent Assoc, v. 104, p. 32-38, 1982.

19. WEINSTEIN, P. Situation-specific child control: a visit to the dentist. Beh Res Ther, v. 34, n. 1, p. 11-21, 1996.
Recebido para publicação em 02/01/00

Enviado para reformulação em 22/03/00

Aceito para publicação em 26/04/00 